\title{
Anisotropy Searches with DAMPE
}

\section{Maria Munoz*}

University of Geneva. Geneva, Switzerland

E-mail: maria.munoz@unige.ch

\section{Mikhail Stolpovskiy}

University of Geneva. Geneva, Switzerland

\section{Shijun Lei}

Purple Mountain Observatory. Nanjing, China

\section{Enrico Catanzani}

University of Perugia. Perugia, Italy

\section{for the DAMPE Collaboration ${ }^{\dagger \ddagger}$}

The DArk Matter Particle Explorer (DAMPE) is a satellite-borne experiment successfully launched in December 2015. The main scientific goal of the mission is to perform high precision measurements of the High Energy Cosmic Ray (HECR) sky and, after more than three years of data taking, DAMPE has collected over 5.6 billion events.

In recent years the anisotropy of CRs results have been presented by several collaborations with the use of ground-based and space-based experiments from energies above tens of $\mathrm{GeV}$ up to EeV. In this work we are present a preliminary study of the sensitivity of the DAMPE experiment to the anisotropy signal. We discuss the method used for anisotropy searches and present the preliminary results based on one year of the DAMPE data for all particle types at calorimeter energies from 100 to $500 \mathrm{GeV}$. The work includes also studies on the different angular scales.

36th International Cosmic Ray Conference -ICRC2019-

July 24th - August 1st, 2019

Madison, WI, U.S.A.

\footnotetext{
*Speaker.

${ }^{\dagger}$ for collaboration list see PoS(ICRC2019)1177

‡The DAMPE mission is funded by the strategic priority science and technology projects in space science of Chinese Academy of Sciences. In China the data analysis is supported in part by the National Key Research and Development Program of China (No. 2016YFA0400200), the National Natural Science Foundation of China (Nos. 11525313, 11622327, 11722328, U1738205, U1738207, U1738208, 11851305), the strategic priority science and tech- nology projects of Chinese Academy of Sciences (No. XDA15051100), the 100 Talents Program of Chinese Academy of Sciences, and the Young Elite Scientists Sponsorship Program. In Europe the activities and the data analysis are supported by the Swiss National Science Foundation (SNSF), Switzerland; the National Institute for Nuclear Physics (INFN), Italy.
} 


\section{Introduction}

The origin, propagation and acceleration of cosmic rays from galactic and extra-galactic sources is currently one the most important open questions in astrophysics. Current theories support that cosmic rays are created in the most extreme environments in space. One of the possible ways to address the question of the origin of the cosmic rays is the study of the anisotropies of their arrival directions. The interstellar medium is filled with the turbulent magnetic fields which make the arrival direction of the cosmic rays highly uniform. The interaction of charged cosmic rays with different magnetic fields modifies their original direction and thus the cosmic rays do not point back to their source. However a strong nearby source can create anisotropy in some direction. Other possible sources of the anisotropy arise from the Compton-Getting effect and diffuse flow of the cosmic rays through the local magnetic fields [3]. Both effects predict that the strongest anisotorpy would be the dipole one and that it might have the amplitude around $\sim 3-5 \times 10^{-4}$ at the energies of order a hundred GeV.

Recent searches for anisotropy have gathered new information in different energy ranges with both space-based and ground-based experiments. Measurements have been made thanks to airshower experiments HAWC [4], MILAGRO [5] and TIBET [6] among others. Searches have also been done with space-based experiments such as PAMELA [7], AMS [8] and FERMI [9, 10, 11]. For Ultra-high Energy Cosmic Rays important results have been presented by the Pierre Auger Observatory [12] and Telescope Array [13].

Sensitivity to the detection of different anisotropies is highly dependent on the type of instrument and its lifetime. Contribution from space-based experiments is expected to be of great interest because they have the full-sky coverage contrary to the partial sky coverage presented for ground experiments. However, an important limitation of space-based experiments is the low rate of events. Considering the energy range of $\sim 100 \mathrm{GeV}$ the most stringent constraint to date on the anisotropies comes from the Fermi-LAT experiment. For the protons with energy higher than 78 $\mathrm{GeV}$ they set a $95 \%$ C.L. upper limit of $1.3 \times 10^{-3}$ on the dipole amplitude [11]. For the electronpositron sample it is $\sim 3 \times 10^{-3}$ at energies above $42 \mathrm{GeV}$ [10]. In this work, we analyse the data collected by the DAMPE satellite experiment [14, 15], using the events collected in the year 2016, in order to search for the all-particle anisotropies, considering events with calorimeter recorded energy in the range from 100 to $500 \mathrm{GeV}$.

\section{The DArk Matter Particle Explorer}

The DArk Matter Particle Explorer (DAMPE) is an space-borne experiment, it was launched the 17th of December 2015 [14, 15]. Since then it has been continuously taking data, orbiting in a sun-synchronous orbit (SSO) at an altitude of $500 \mathrm{~km}$, each orbit has a duration of 95 minutes. DAMPE's main objective is the measure of cosmic rays from $20 \mathrm{GeV}$ to the $\mathrm{TeV}$ regime. DAMPE consists of four sub-detectors, from top to bottom these are:

- The Plastic Scintillator Detector (PSD) is a double layer array of plastic strips that covers the top of the instrument, for charge measurements and as anti-coincidence shield for $\gamma$-rays. 
- The Silicon tungsten tracker (STK) is composed of six double-layers of silicon strips, for measuring the incoming direction of the high energy particles.

- The Bismuth Germanium Oxide (BGO) calorimeter is composed of 308 BGO scintillating bars, arranged in 14 layers. Each bar has two PMTs at both ends of the bars for read out. $\mathrm{BGO}$ has a depth of $\sim 32$ radiations lengths allowing high energy resolution.

- The Neutron Detector (NUD) consists of four blocks of boron-loaded plastics scintillators wrapped in aluminum film, used for hadrons identification for energies above $150 \mathrm{GeV}$.

DAMPE observes the full sky twice in a year. Its angular resolution is better than $0.2^{\circ}$ at $100 \mathrm{GeV}$. The instantaneous field of view of the instrument is approximately $1 \mathrm{sr}$. Thanks to its thick BGO calorimeter DAMPE has an excellent energy resolution and energy reach for the direct measurement of cosmic rays, such qualities make DAMPE a competitive instrument for the anisotropy search.

\section{Data Set}

The analyzed data set consists of data collected in 2016. Selection cuts are applied to assure that the particle track and shower are fully contained in the detector volume. These cuts include a maximum $60^{\circ}$ cut on the zenith angle in the detector coordinates. Events detected while the detector is crossing the South Atlantic Anomaly are rejected.

Since we use the all-particle data set, for the sake of consistency we apply no correction to the raw BGO energy measurement. For example for a proton at $800 \mathrm{GeV}$ on average the calorimeter contains only about $40 \%$ of hadronic shower. Thus the energies plotted on all the figures below are factor $\sim 1.5-2$ lower than the actual particle energies. This energy correction factor varies from one particle type to another. That is why it was decided to use the raw BGO energy instead. Note also, that the Fermi results mentioned above are for the corrected (true) particle energy.

Although DAMPE is able to identify types of the incoming particles, such identification requires quite stringent selection cuts. For example, for the submitted paper on the proton flux [2] the number of selected events is about $2 \times 10^{6}$ for a 30 month period. With the all-particle sample one can have approximately 6-7 times more data. It is the count statistics which has the strongest impact on the anisotropy sensitivity. That is why we decide to use the all-particle sample, conforming with the ground-based experiments operating on higher energies than DAMPE.

The all-particle sample, according to the flux studies [1,2], consists of protons on $\sim 90 \%$. The BGO energy of $100 \mathrm{GeV}$ for a proton roughly corresponds to the true particle energy of $200 \mathrm{GeV}$. However, we do not suggest to interpret the results presented in this work as the proton anisotropy at twice higher energies. Such an interpretation would require extensive study of the systematics with $\sim 10 \%$ contamination of other particle types. Instead, we suggest the consideration in light of the Compton-Getting effect [16] and diffuse anisotropy [17], which both could be studied in the all-particle sample.

On BGO energy we set a minimum energy cut of $100 \mathrm{GeV}$ in order to reject the low energy events for which the Earth magnetic field effects could be quite strong. For the current study we limit the maximum BGO energy at $500 \mathrm{GeV}$. 
After all cuts the total amount of events for the analysis is about $6.1 \times 10^{6}$. The distribution of the selected events as a function of energy is showed in Figure 1.

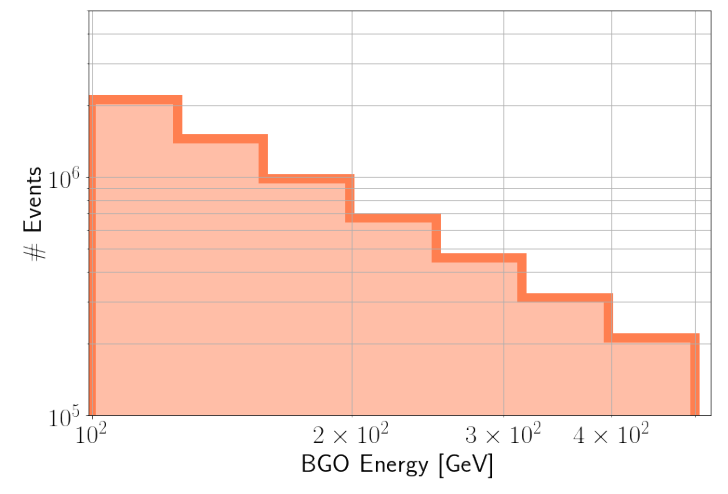

Figure 1: Distribution of the number of events as a function of the raw BGO energy for the all particle selection used for this work. Energy range from $100 \mathrm{GeV}$ to $500 \mathrm{GeV}$. The total amount of events collected for 1 years of measurements is $\sim 6.1 \times 10^{6}$.

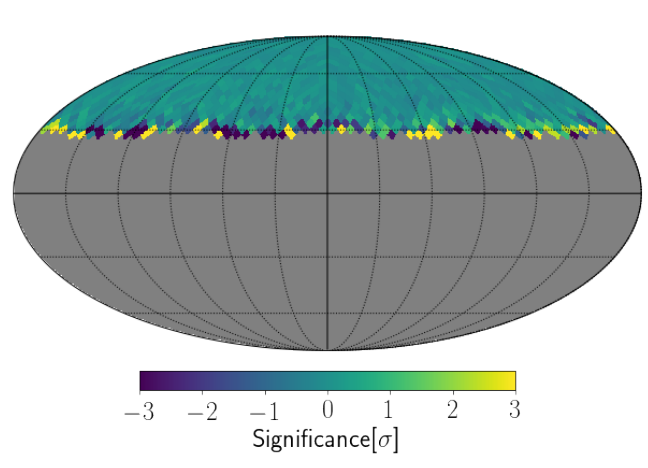

Figure 2: Sky map in altitude-azimuth coordinates. The East-west effect is not visible on our data set thanks to the 60 degree imposed cut on the data. Map done using healpix with nside $=16$.

The so-called East-West effect arises from the fact that the cosmic ray trajectories are bent in the presence of the Earth magnetic field. Thus the positively charged cosmic rays coming from the East of the satellite could blocked by the Earth. Hence there are less events detected on the East side of the instrument than on the West side. This effect is particularly strong at large values of the zenith angle and at low energies. We check that the East-West effect is not visible in our data. The significance map in altitude-azimuth coordinates is shown on the figure 2 . As one can see, the $60^{\circ}$ quality cut on zenith also makes sure that the East-West effect is avoided.

\section{Anisotropy Search}

For this work we will focus on anisotropy searches at large angular scales. As it was mentioned before, the center of interest is the dipole anisotropy with an expected amplitude $O\left(10^{-4}\right)$. If the sky coverage was uniform it would be possible to study the anisotropies directly from the sky map $D$ which in each pixel contains the number of entries in that pixel.

However, the DAMPE sky coverage is not uniform. Therefore it is necessary to create the exposure map $R$. We create reference maps with the rate-based method explained in [10] with the orbit divided in $1 \mathrm{~s}$ intervals. The exposure map is then estimated as an average 100 reference maps made with this method. The exposure map of DAMPE is shown on the figure 3

Once one has the observation map $D$ and the exposure map $R$, the relative intensity is defined as follows:

$$
\delta I=\frac{D}{R}-1
$$

The reconstructed relative intensity map $\delta I$ is shown on the figure 4 . 


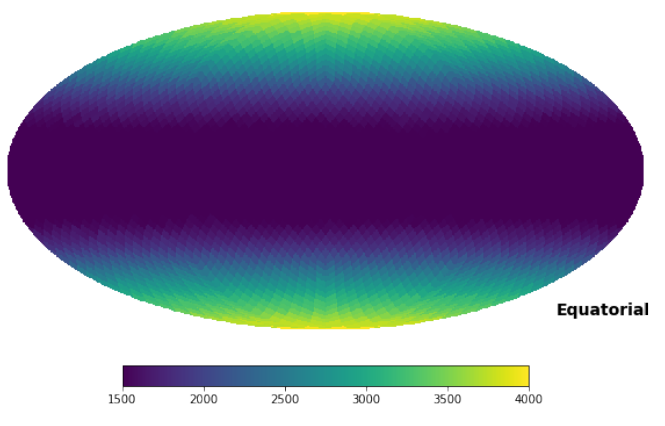

Figure 3: Exposure map, plotted with nside $=$ 16 in the equatorial coordinates.

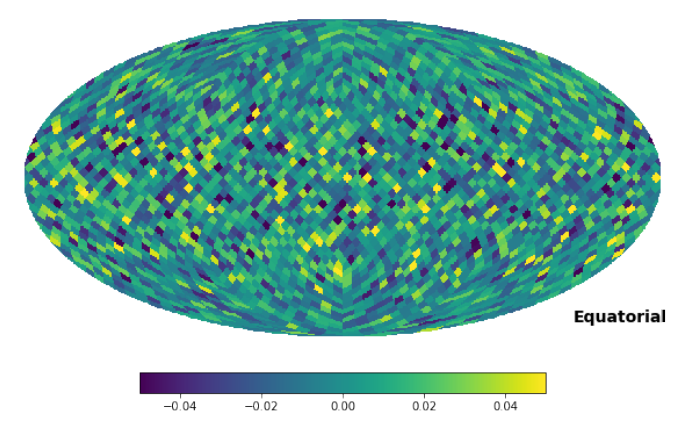

Figure 4: Relative intensity map, plotted with $n s i d e=16$ in the equatorial coordinates.

\subsection{Spherical Harmonics}

To analyse the relative intensity map we use the spherical harmonic analysis and estimate the angular power spectrum of the observed anisotropies:

$$
\hat{C}_{\ell}=C_{\ell}-C_{N},
$$

where $C_{\ell}$ is the reconstructed power spectrum calculated from the coefficients $a_{\ell m}$ of the spherical harmonic decomposition of $\delta I$ and $C_{N}$ is the noise contribution. By definition:

$$
C_{\ell}=\frac{1}{2 \ell+1} \sum_{m=-\ell}^{\ell}\left|a_{\ell m}\right|^{2} .
$$

In order to compute the power spectrum $C_{\ell}$ we use the HEALPix package [18]. The noise contribution $C_{N}$ is estimated as:

$$
C_{N}=\frac{4 \pi}{N_{p i x}^{2}} \sum_{i=1}^{N_{p i x}} \frac{N_{i}}{<N_{i}>^{2}} .
$$

Here $N_{\text {pix }}$ is the number of pixels on the map and $N_{i}$ is the number of entries at the $i$-th pixel [10]. The uncertainty of the measured $C_{\ell}$ is defined, following [19], as:

$$
\delta C_{\ell}=\sqrt{\frac{2}{2 \ell+1}} C_{\ell}
$$

where we neglect the statistical term, which is several orders of magnitude smaller than the sample variance term expressed above. The power spectrum of the observed anisotropies $\delta I$ at the lowest energy bin is shown on the figure 5 .

As been said, we are particularly interested in the dipole anisotropy. which is defined as:

$$
\delta=3 \sqrt{\frac{C_{1}}{4 \pi}}
$$

where $C_{1}$ is the value of the angular power spectrum at $\ell=1$. The distribution of expected results under the null hypothesis (i. e. no anisotropy) is calculated by running many sky simulations with no input anisotropy and reconstructing the dipole amplitude from those maps. 


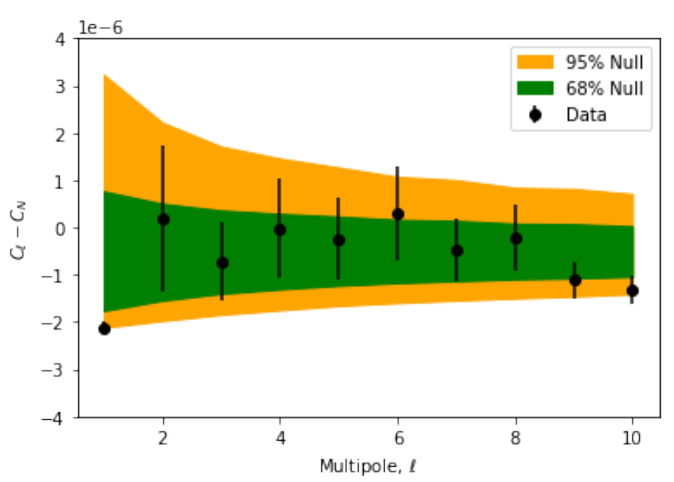

Figure 5: Estimator of the anisotropy angular power spectrum, according to the equation 4.2. Data points are shown in black. The green and orange bands show correspondingly the $68 \%$ and $95 \%$ limits calculated with the null hypothesis (i. e. perfect isotropy).

\section{Results}

After reconstructing the angular power spectra of the observed map we are able to compute the dipole amplitude using the equation 4.6. Its value is $(3.3 \pm 1.3) \times 10^{-4}$, while the distribution of the null-hypothesis results has median at $1.1 \times 10^{-3}$ with an interval comprising $68 \%$ of the probability density function from $6.7 \times 10^{-4}$ to $1.7 \times 10^{-3}$. One can clearly see that the reconstructed dipole amplitude lies even below the null-hypothesis $1 \sigma$ range, which means that with the analysed statistics DAMPE is not able to claim the detection of the dipole anisotropy.

The 95\% C.L. upper limits on the dipole amplitude are derived as following: if the true dipole component of the power spectrum is equal $\tilde{C}_{1}=\hat{C}_{1}+C_{N}$, then the probability to measure $C_{1}$ is [10]:

$$
P\left(C_{1} \mid \hat{C}_{1}, C_{N}\right)=\frac{3 \sqrt{3}}{\sqrt{2 \pi} \tilde{C}_{1}} \sqrt{\frac{C_{1}}{\tilde{C}_{1}}} \exp \left(-\frac{3 C_{1}}{2 \tilde{C}_{1}}\right) .
$$

The posterior probability is given by:

$$
P\left(\hat{C}_{1} \mid C_{1}, C_{N}\right) \propto \frac{P\left(C_{1} \mid \hat{C}_{1}, C_{N}\right)}{\hat{C}_{1}+C_{N}}
$$

and using the Bayesian approach the upper limit corresponds to the solution of:

$$
\int_{0}^{C_{1}^{u . l .}} P\left(\hat{C}_{1} \mid C_{1}, C_{N}\right) d \hat{C}_{1}=1-C . L .
$$

The corresponding limit for the dipole amplitude is calculated from $C_{1}^{u . l .}$ using equation 4.6. The 95\% confidence level upper limit on $\delta$ calculated from the median of the distribution of $C_{1}$ under the null hypothesis is equal to $4.0 \times 10^{-3}$.

\section{Conclusions and future scope}

In this work we report on the analysis of one year data set of DAMPE for the search of the arrival direction anisotropies of the cosmic rays independent of the type of the particle (all-particle 
sample). The total number of events is $6.1 \times 10^{6}$ and the $95 \%$ C. L. upper limit on the dipole amplitude is $4.0 \times 10^{-3}$.

Since the sensitivity for the anisotropies improves as a square root of the available number of events we can expect to reach the level of $2.1 \times 10^{-3}$ analysing the full DAMPE data set. We are also working to increase the data sample both by loosing the selection cuts and accepting events outside the BGO fiducial volume but still with a good angular resolution, with the goal to increase the total number of events by a factor up to 3 .

\section{References}

[1] Ambrosi, G., An, Q., Asfandiyarov, R., Azzarello, P., Bernardini, P., Bertucci, B., ... Chen, J. L. (2017). Direct detection of a break in the teraelectronvolt cosmic-ray spectrum of electrons and positrons. Nature, 552(7683), 63.

[2] C. Yue, J. Zang, T. Dong, A. Surdo, S. Vitillo on behalf of the DAMPE Collaboration, "Studies of Cosmic-Ray Proton Flux with the DAMPE Experiment", PoS(ICRC2017)1076

[3] Ahlers, M., Mertsch, P. (2017). Origin of small-scale anisotropies in Galactic cosmic rays. Progress in Particle and Nuclear Physics, 94, 184-216.

[4] HAWC, A.U. Abeysekara et al., ApJ 796 (2014) 108, 1408.4805.

[5] Abdo, A. A., et al. "The large-scale cosmic-ray anisotropy as observed with Milagro." The Astrophysical Journal 698.2 (2009): 2121.

[6] M. Amenomori et al., Astrophys. J. 711 (2010) 119, 1001.2646.

[7] Adriani, O., Barbarino, G. C., Bazilevskaya, G. A., Bellotti, R., Boezio, M., Bogomolov, E. A., ... Cafagna, F. (2015). Search for anisotropies in cosmic-ray positrons detected by the PAMELA experiment. The Astrophysical Journal, 811(1), 21.

[8] La Vacca, G. (2016). Search for Cosmic Ray Anisotropy with the Alpha Magnetic Spectrometer on the International Space Station. arXiv preprint arXiv:1612.08957.

[9] Ackermann, Markus, et al. "Searches for cosmic-ray electron anisotropies with the Fermi Large Area Telescope." Physical Review D 82.9 (2010): 092003.

[10] Abdollahi, S., et al. "Search for cosmic-ray electron and positron anisotropies with seven years of Fermi Large Area Telescope data." Physical review letters 118.9 (2017): 091103.

[11] Ajello, M., Baldini, L., Barbiellini, G., Bastieri, D., Bechtol, K., Bellazzini, R., ... Brandt, T. J. (2019). A Search for Cosmic-ray Proton Anisotropy with the Fermi Large Area Telescope. ISO 690

[12] Aab, A., Abreu, P., Aglietta, M., Al Samarai, I., Albuquerque, I. F. M., Allekotte, I., ... Anchordoqui, L. (2017). Observation of a large-scale anisotropy in the arrival directions of cosmic rays above $8 \times 10^{18} \mathrm{eV}$. Science, 357(6357), 1266-1270.

[13] T. Abu-Zayyad et al. Search for Anisotropy of Ultra- High Energy Cosmic Rays with the Telescope Array Experiment. Astrophys. J., 757:26, 2012. arXiv:1205.5984, doi:10.1088/0004-637X/757/1/26.

[14] J. Chang et al. [DAMPE Collaboration], "The DArk Matter Particle Explorer mission," arXiv:1706.08453 [astro-ph.IM].

[15] Ambrosi, G., et al. "The on-orbit calibration of DArk Matter Particle Explorer." Astroparticle Physics 106 (2019): 18-34. 
[16] A.H. Compton and I.A. Getting, Physical Review 47 (1935) 817.

[17] Jones, Frank C. "The generalized diffusion-convection equation." The Astrophysical Journal 361 (1990): 162-172.

[18] Gorski, K. M., Hivon, E., Banday, A. J., et al. 2005, The Astrophysical Journal, 622, 759. http://stacks.iop.org/0004-637X/622/i=2/a=759

[19] Knox, LLoyd. "Determination of inflationary observables by cosmic microwave background anisotropy experiments." Physical Review D 52.8 (1995): 4307. 